Edilson Almeida de Oliveira'

Andréa Dâmaso Bertoldi"

Marlos Rodrigues Domingues"I

Iná S Santos"

Aluísio J D Barros"

Programa de Pós-Graduação em Saúde Coletiva. Universidade do Vale do Rio dos Sinos. São Leopoldo, RS, Brasil

" Departamento de Medicina Social. Faculdade de Medicina. Universidade Federal de Pelotas (UFPel). Pelotas, RS, Brasil

III Departamento de Desportos. Escola Superior de Educação Física. UFPel. Pelotas, RS, Brasil

Correspondence:

Edilson Almeida de Oliveira

R. Estácio de Sá, 1073

Residencial Itaperoá, Apto 703

Vila Bosque

87005-020 Maringá, PR, Brasil

E-mail: proedilson@ibest.com.br

\section{Factors associated to medicine use among children from the 2004 Pelotas Birth Cohort (Brazil)}

\author{
Fatores associados ao uso de \\ medicamentos na coorte de \\ nascimentos de Pelotas 2004
}

\begin{abstract}
OBJECTIVE: To identify factors associated to medicine use among children from the 2004 Pelotas Birth Cohort, Brazil.

METHODS: Prospective study to evaluate medicine use in children aged 3, 12 and 24 months regardless of the reasons, therapeutic indication or class. The study included 3,985 children followed up at three months of age, 3,907 at 12 months, and 3,868 at the last follow-up time of 24 months. Mothers were interviewed to collect information on medicine use during the recall period of 15 days prior to the interview. The outcome was studied according to sociodemographic and perinatal variables, mother's perception of child's health and breastfeeding status. Crude and adjusted analyses were performed by Poisson regression following a hierarchical model.
\end{abstract}

RESULTS: The prevalence of medicine use ranged from $55 \%$ to $65 \%$ in the three follow-ups. After controlling for confounders, some variables remained associated to medicine use only at the three-month follow-up with greatest use among children of younger mothers, those children who had intrapartum complications, low birthweight, were never breastfed and were admitted to a hospital. Greatest medicine use was also associated with being a firstborn child at 3 and 12 months; mother's perception of their child health as fair or poor and children whose mothers have private health insurance at 12 and 24 months; highest maternal education level at all follow-up times.

CONCLUSIONS: Different variables influence medicine use among children during the first two years of life and they change as the child ages especially maternal factors and those associated to the child's health problems.

DESCRIPTORS: Infant. Child. Drug Utilization. Risk Factors. Maternal and Child Health. Cohort Studies. 


\section{RESUMO}

OBJETIVO: Identificar fatores associados ao uso de medicamentos nas crianças da coorte de nascimentos de 2004 de Pelotas, RS.

MÉTODOS: Estudo de delineamento prospectivo, utilizando como desfecho o uso de medicamentos pelas crianças aos três, 12 e 24 meses (independentemente do motivo, indicação ou grupo terapêutico). O estudo inclui 3.985 crianças no seguimento aos três meses, 3.907 no de 12 meses e 3.868 no último seguimento aos 24 meses. Foi realizada entrevista com as mães, referente ao uso de medicamento durante período recordatório de 15 dias anteriores. O desfecho foi analisado de acordo com variáveis sociodemográficas, perinatais, da percepção materna de saúde da criança e de amamentação. Foram realizadas análises bruta e ajustada utilizando regressão de Poisson e seguindo um modelo hierarquizado de análise.

RESULTADOS: A prevalência do uso de medicamentos variou entre 55\% e 65\% nos três seguimentos. Após análise ajustada, algumas variáveis permaneceram associadas ao uso de medicamentos apenas no acompanhamento dos três meses, com maior utilização entre as crianças com mães mais jovens, quando houve algum problema de saúde no momento do parto, baixo peso ao nascer, nas crianças que nunca mamaram e quando houve internação hospitalar da criança. Também se associou ao maior uso de medicamentos: aos três e 12 meses o fato de ser primogênito; aos 12 e 24 meses a percepção de saúde da criança pela mãe como regular ou ruim e o fato de a mãe possuir plano de saúde; $\mathrm{e}$ nos três acompanhamentos, a maior escolaridade das mães.

CONCLUSÕES: Variáveis diferentes influenciam a utilização de medicamentos nos primeiros dois anos de vida em função do avanço da idade da criança, sobretudo fatores associados à mãe da criança e a problemas de saúde da criança.

\section{DESCRITORES: Lactente. Criança. Uso de Medicamentos. Fatores de Risco. Saúde Materno-Infantil. Estudos de Coortes.}

\section{INTRODUCTION}

Medicine use among children is affected by many factors that change widely, and frequently social concerns outweigh medical reasons. ${ }^{22}$ Medicines play a major role in health and understanding use patterns to prevent irrational use is a major goal of pharmacoepidemiology. ${ }^{1}$

Studies on medicine use among children carried out in the 1980s and 1990s in the UK showed that most mothers $(96 \%)$ reported their children used some medicine during their first six months of life. ${ }^{17}$ A study from the Netherlands reported that nearly $10 \%$ of children used at least one medicine in the first month of life and, by the age of two, the proportion increased to $81 \% .^{25}$ In Southern Brazil, an epidemiological study showed that medicine use during the first three months of life is very frequent with a prevalence higher than $65 \%$ and long-term use (one month or longer) was reported by $20 \%$ of mothers. The factors associated to higher medicine use included: high income; firstborn child; high number of medical visits; hospitalization; and maternal education level between five to eight years of schooling. Higher medicine use was also seen among children who were not breastfed. ${ }^{28}$

Different factors related to family, community and health settings influence medicine use. At the family level, the perception of the need of medicines, conceptions about efficacy (or lack thereof), prices and education level contribute to medicine use patterns. ${ }^{16}$ Medicine use in children are associated to sociodemographic characteristics, health insurance status, child's age younger than five, medical visits in the past days, mothers' perception of their child's health and health conditions..$^{12,15,21}$

The present study aimed to identify factors associated to medicine use among children from the 2004 Pelotas Birth Cohort, Brazil. 


\section{METHODS}

The study was based on data from the 2004 Pelotas Birth Cohort, Brazil, the third birth cohort study conducted in the city of Pelotas, southern Brazil. All children born during 2004 in the city's urban area and Jardim América district (municipality of Capão do Leão) were enrolled. At the perinatal follow-up all information were obtained from the child's mother. In subsequent stages the mother was chiefly the respondent but the child's father or carer were sometimes the respondents. More methodological details of the study can be found elsewhere. ${ }^{5,6}$

The study outcome was report of medicine use regardless of therapeutic indication or class at the three, 12and 24-month follow-up during a 15-day recall period. Mothers were asked about their children's medicine use in the past 15 days and then medicine names were recorded and prescriptions and packages requested.

The outcomes were analyzed according to demographic, socioeconomic and perinatal variables along with breastfeeding status and mother's perception of their child's health. Sociodemographic information collected were: gender, maternal skin color, maternal age (complete years), socioeconomic condition (based on the National Economic Index [IEN] for the city of Pelotas, in quintiles, where the first quintile represents the poorest $20 \%$ of the population), maternal education level (complete years of schooling), maternal and child's private health insurance (at the age of 12 and 24 months). The IEN is an index based on 12 asset items and schooling of the head of the family. The index was developed based on a principal component analysis using variables from the 2000 Population Census of the Instituto Brasileiro de Geografia e Estatística (Brazilian Institute of Geography and Statistics). ${ }^{4}$

The perinatal variables included: intrapartum complications; neonatal intensive care unit (ICU) admission; low birthweight $(<2500 \mathrm{~g})$; preterm birth (according to an algorithm combining the last menstrual period, ultrasound scan or the Dubowitz method); being a firstborn child, type of delivery (vaginal or cesarean); child's hospitalization soon after delivery; and gestational morbidity (hypertension, diabetes, depression, threatened miscarriage, bleeding, vaginal discharge, urinary tract infection or other infections during pregnancy, according to maternal report). Anemia was not included as a gestational morbidity because of its high prevalence among pregnant women, which would distort the prevalence of the variable as a whole.

Mother's perception of their child's health was assessed at the follow-ups of 12 and 24 months. Although this information is available for the follow-up at 3 months, it was not included in the analysis because data was only collected in a subsample. Breastfeeding information (in months) was also assessed and was categorized at three months as never breastfed, quit breastfeeding or currently breastfeeding. At the ages of 12 and 24 months the categories were: never breastfed, breastfed until the age of three months, breastfed up to six months of age, and breastfed longer than six months.

Data was entered in EpiInfo $6.0^{13}$ and analyses were performed in STATA 9.0. First, crude analyses were carried out (outcomes and independent variables) to determine prevalence ratios and $95 \%$ confidence intervals. Wald's tests for heterogeneity and linear trend were used to measure associations. Then, a multivariable analyses (Poisson regression) was carried out, ${ }^{3}$ following a hierarchical model (Figure). ${ }^{27}$ The Poisson regression aims at controlling for confounders, retaining in the model variables presenting association between $5 \%$ and $20 \%$. All variables are controlled for the same hierarchical level and upper levels.

All phases of the study were approved by the Research Ethics Committee of the Medical School of Universidade Federal de Pelotas (protocol nr. 4.06.00.006, approved on 20 June 2006 and protocol nr. 4.06.01.113, approved on 9 March 2005). All interviews and examinations were only performed after parents or carers signed a written consent form.

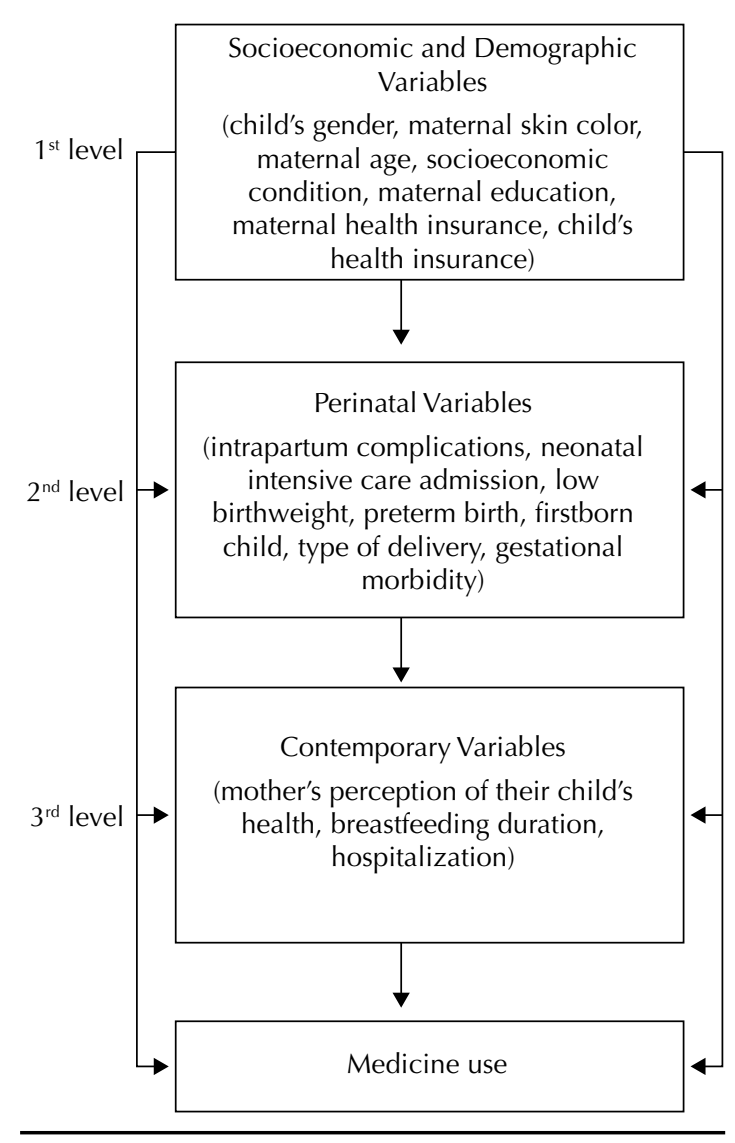

Figure. Hierarchical model of analysis of medication use in children. 2004 Pelotas Birth Cohort, Southern Brazil. 


\section{RESULTS}

Of 4,231 live births enrolled in the 2004 Pelotas Birth Cohort, the present study included 3,985 children followed up at three months of age, 3,907 at 12 months, and 3,868 at the last follow-up time of 24 months. Excluding deaths, follow-up rates were 95.7\%, 94.3\% and $93.5 \%$, respectively. From the beginning of the study up to the 24-month visit, loss and refusal rate was $6.5 \%$ (274 children).

The prevalence of medicine use in children ranged from $55 \%$ to $65 \%$ : $65 \%$ at three months (95\% CI 63.5;66.5); $64.4 \%$ at 12 months (95\% CI 63.5;66.5); and 54.7\% at 24 months (95\% CI 53.1;56.2).

Of the 4,231 children from the 2004 Birth Cohort, most were male, and their mothers were mostly white, 20-29 years old with less than eight years of schooling. Around two-thirds of the children did not have health insurance, $13 \%$ had intrapartum complications, $6 \%$ were admitted to a neonatal intensive care unit (ICU), $10 \%$ were low birthweight, $19 \%$ were hospitalized during the first year of life and $40 \%$ were firstborn. Most mothers had gestational morbidities, perceived their child's health as "excellent" and 81\% attended public hospitals.

The highest rates of medicine use were seen among firstborn children, delivered by C-section, who were admitted to neonatal ICU, born to more educated, better-off and white women with health insurance. The prevalence ratios are presented in Table 1.

Socioeconomic condition was associated to medicine use at all follow-up times in the bivariate analysis, but after adjustment the association was no longer significant (Table 2).

At three months, the adjusted analysis showed $9 \%$ higher medicine use among children from white-skin $(p=0.04)$ compared to black-skin mothers; children from younger mothers (12-19 years) had a $13 \%$ increased use $(\mathrm{p}=0.01)$ compared to children from older mothers (36 years or older). Maternal education level (12 years or more) was associated to a $7 \%$ increase in medicine use $(\mathrm{p}=0.04)$; intrapartum complications was associated to $10 \%$ increase $(\mathrm{p}=0.009)$; low birthweight to $11 \%$ increase $(\mathrm{p}=0.01)$; firstborn child $10 \%$ increase $(p=0.001)$; gestational morbidity and hospitalization $16 \%$ and $26 \%$ increase, respectively, $(\mathrm{p}<0.001)$. Breastfeeding was also associated with higher medicine use $(\mathrm{p}<0.001)$; children who were never breastfed used $25 \%$ more medicines compared to those still breastfeeding at three months (Table 2).

After the adjusted analysis we found that 12-month-old boys had $3 \%$ higher medicine use $(p=0.03)$; maternal education level had a borderline association $(p=0.05)$; maternal health insurance was associated $(p=0.02)$ as well as child's health insurance $(p=0.006)$ with a $9 \%$ increase in medicine use. Being a firstborn child remained associated $(\mathrm{p}<0.001)$ to a $10 \%$ increase in use; as well as mother's perception of the child's health $(p<0.001)$. The remaining variables that were associated at three months were no longer associated at 12 months (Table 2).

After the adjusted analysis, medicine use was higher among 24-month-old children from more educated women $(\mathrm{p}<0.001)$, the only variable that was associated with medicine use at all three follow-up times, and was more evident at the follow-up of 24 months. Children from mothers with 12 or more years of schooling had a 34\% higher medicine use compared to mothers with lower education; children from mothers with health insurance were $13 \%$ more likely $(\mathrm{p}<0.001)$ to use medicine at 24 months; neonatal ICU admission was also associated to higher $(16 \%)$ medicine use $(p=0.01)$ as well as $\mathrm{C}$-section ( $9 \%$ increase; $\mathrm{p}=0.007)$. Type of delivery was only associated to medicine use at 24 months; gestational morbidity that was not associated at 12 months showed a significant $(p=0.006) 12 \%$ increase in medicine use; and mother's perception of the child's health was strongly associated to the outcome $(p<0.001)$. The mother's perception of the child's health was a strong predictor at 12 and 24 months and was associated to increased medicine use when health was rated as fair $(52 \%)$ or poor $(74 \%)$ compared to excellent health (Table 2).

\section{DISCUSSION}

Cohort studies allow us to understand and to establish chronologically facts that are being studied, and to observe early characteristics that may influence medicine use. ${ }^{28}$

In our study there was a reduction in the prevalence of medicine use over the three follow-up times, especially at 24 months. Similar results were found in a 2005 Italian study (Lombardia) reporting a prevalence of $65 \%$ among children from 12 months to five years of age. ${ }^{15}$ In the 1982 Pelotas Birth Cohort, the prevalence of medicine use was $56 \%$ in children aged 35 to 53 months. ${ }^{9}$

Medicine use throughout the life course shows a J-shaped distribution, that is, higher prevalence during early life, decreasing during adolescence and gradually increasing in adult life with the highest prevalences seen among the elderly. ${ }^{2}$

The factors associated to medicine use in children up to two years of age were different from those described in adults. Among adults there is a large gender-difference, with higher use among women. ${ }^{10,23}$ In the present study, gender was not a determinant of medicine use. Only at 12 months a slightly higher use was observed among boys. Another difference between child and adult use is the socioeconomic influence. In adults socioeconomic condition is a major determinant of medicine use and many times it outweighs the actual need with a higher 
Table 1. Prevalence and crude analysis of factors associated to medicine use at 3, 12 and 24 months of age. 2004 Pelotas Birth Cohort, Southern Brazil.

\begin{tabular}{|c|c|c|c|c|c|c|c|c|c|}
\hline \multirow[b]{2}{*}{ Variable } & \multicolumn{3}{|c|}{3 months } & \multicolumn{3}{|c|}{12 months } & \multicolumn{3}{|c|}{24 months } \\
\hline & $\begin{array}{c}\mathrm{P} \\
(\%)\end{array}$ & PR $(95 \% \mathrm{Cl})$ & p-value & $\begin{array}{c}\mathrm{P} \\
(\%)\end{array}$ & PR $(95 \% \mathrm{Cl})$ & $p$-value & $\begin{array}{l}\mathrm{P} \\
(\%)\end{array}$ & PR $(95 \% \mathrm{Cl})$ & $\mathrm{p}$-value \\
\hline Gender & & & $0.7^{*}$ & & & $0.04^{*}$ & & & $0.2^{*}$ \\
\hline Male & 64.7 & $0.99(0.95 ; 1.04)$ & & 65.9 & $1.05(1.00 ; 1.10)$ & & 55.6 & $1.04(0.98 ; 1.10)$ & \\
\hline Female & 65.4 & 1 & & 62.8 & 1 & & 53.6 & 1 & \\
\hline $\begin{array}{l}\text { Maternal skin color } \\
\text { (observed by interviewer) }\end{array}$ & & & $<0.001^{*}$ & & & $0.002^{*}$ & & & $<0.001^{*}$ \\
\hline White & 66.8 & $1.13(1.06 ; 1.20)$ & & 66.0 & $1.08(1.02 ; 1.15)$ & & 56.6 & $1.17(1.08 ; 1.27)$ & \\
\hline Black & 59.1 & 1 & & 61.1 & 1 & & 48.3 & 1 & \\
\hline Other & 63.6 & $1.07(0.97 ; 1.20)$ & & 56.5 & $0.92(0.82 ; 1.04)$ & & 52.8 & $1.09(0.96 ; 1.25)$ & \\
\hline Maternal age (years) & & & $0.2^{* *}$ & & & $0.7^{* *}$ & & & $0.4^{*}$ \\
\hline $12-19$ & 66.4 & $1.08(0.98 ; 1.18)$ & & 63.0 & $1.08(0.97 ; 1.19)$ & & 52.7 & $1.01(0.90 ; 1.14)$ & \\
\hline $20-29$ & 65.2 & $1.07(0.98 ; 1.16)$ & & 64.6 & $1.10(1.01 ; 1.20)$ & & 55.3 & $1.06(0.96 ; 1.18)$ & \\
\hline $30-35$ & 65.5 & $1.09(0.99 ; 1.20)$ & & 68.0 & $1.16(1.06 ; 1.28)$ & & 56.0 & $1.08(0.96 ; 1.20)$ & \\
\hline$\geq 36$ & 61.0 & 1 & & 58.6 & 1 & & 52.1 & 1 & \\
\hline $\begin{array}{l}\text { Socioeconomic condition } \\
\text { (quintiles of IEN) }\end{array}$ & & & $<0.001$ ** & & & $<0.001$ * * & & & $<0.001$ ** \\
\hline $1 \mathrm{st}$ & 58.1 & 1 & & 56.0 & 1 & & 46.4 & 1 & \\
\hline 2nd & 65.9 & $1.13(1.05 ; 1.22)$ & & 64.3 & $1.15(1.06 ; 1.24)$ & & 54.1 & $1.17(1.06 ; 1.28)$ & \\
\hline $3 \mathrm{rd}$ & 66.4 & $1.14(1.06 ; 1.23)$ & & 64.6 & $1.15(1.07 ; 1.25)$ & & 56.4 & $1.21(1.11 ; 1.33)$ & \\
\hline 4th & 67.7 & $1.17(1.08 ; 1.26)$ & & 68.4 & $1.22(1.13 ; 1.32)$ & & 58.0 & $1.25(1.13 ; 1.38)$ & \\
\hline 5 th & 69.5 & $1.20(1.11 ; 1.29)$ & & 72.4 & $1.29(1.20 ; 1.39)$ & & 61.0 & $1.31(1.20 ; 1.44)$ & \\
\hline $\begin{array}{l}\text { Maternal education } \\
\text { (complete years of } \\
\text { schooling) }\end{array}$ & & & $<0.001^{\text {* * }}$ & & & $<0.001^{* *}$ & & & $<0.001$ * * \\
\hline $0-4$ & 59.5 & 1 & & 57.5 & 1 & & 43.0 & 1 & \\
\hline $5-8$ & 62.7 & $1.05(0.98 ; 1.14)$ & & 61.3 & $1.07(0.99 ; 1.16)$ & & 53.2 & $1.24(1.11 ; 1.37)$ & \\
\hline $9-11$ & 69.3 & $1.16(1.08 ; 1.25)$ & & 68.3 & $1.19(1.10 ; 1.29)$ & & 59.1 & $1.37(1.24 ; 1.52)$ & \\
\hline$\geq 12$ & 68.4 & $1.15(1.05 ; 1.26)$ & & 73.8 & $1.28(1.17 ; 1.41)$ & & 63.9 & $1.48(1.32 ; 1.67)$ & \\
\hline $\begin{array}{l}\text { Mother with health } \\
\text { insurance }\end{array}$ & & & $<0.001^{*}$ & & & $<0.001^{*}$ & & & $<0.001^{*}$ \\
\hline Yes & 69.4 & $1.11(1.06 ; 1.16)$ & & 72.5 & $1.20(1.15 ; 1.26)$ & & 62.1 & $1.22(1.15 ; 1.29)$ & \\
\hline No & 62.8 & 1 & & 60.4 & 1 & & 50.9 & 1 & \\
\hline $\begin{array}{l}\text { Child with health } \\
\text { insurance }\end{array}$ & & & - & & & $<0.001^{\star}$ & & & $<0.001^{\star}$ \\
\hline Yes & - & & & 71.9 & $1.19(1.14 ; 1.25)$ & & 61.0 & $1.19(1.12 ; 1.26)$ & \\
\hline No & - & & & 60.2 & 1 & & 50.8 & 1 & \\
\hline Intrapartum complications & & & $<0.001^{*}$ & & & $0.02 *$ & & & $0.05^{*}$ \\
\hline Yes & 73.2 & $1.15(1.08 ; 1.22)$ & & 69.2 & $1.09(1.02 ; 1.16)$ & & 58.7 & $1.09(1.00 ; 1.18)$ & \\
\hline No & 63.9 & 1 & & 63.7 & 1 & & 54.1 & 1 & \\
\hline Neonatal ICU admission & & & $<0.001^{*}$ & & & $0.02 *$ & & & $0.002^{*}$ \\
\hline Yes & 80.3 & $1.25(1.16 ; 1.34)$ & & 71.1 & $1.11(1.01 ; 1.22)$ & & 64.1 & $1.18(1.06 ; 1.32)$ & \\
\hline No & 64.2 & 1 & & 64.0 & 1 & & 54.1 & 1 & \\
\hline Low birthweight $(<2500 \mathrm{~g})$ & & & $<0.001^{*}$ & & & $0.02 *$ & & & $0.8^{*}$ \\
\hline Yes & 74.8 & $1.17(1.09 ; 1.25)$ & & 69.6 & $1.09(1.01 ; 1.17)$ & & 54.0 & $1.01(0.91 ; 1.12)$ & \\
\hline No & 64.0 & 1 & & 63.9 & 1 & & 54.7 & 1 & \\
\hline
\end{tabular}

To be continued 
Table 1 continuation

\begin{tabular}{|c|c|c|c|c|c|c|c|c|c|}
\hline \multirow[b]{2}{*}{ Variable } & \multicolumn{3}{|c|}{3 months } & \multicolumn{3}{|c|}{12 months } & \multicolumn{3}{|c|}{24 months } \\
\hline & $\begin{array}{c}\mathrm{P} \\
(\%)\end{array}$ & PR $(95 \% \mathrm{Cl})$ & $p$-value & $\begin{array}{c}\mathrm{P} \\
(\%)\end{array}$ & PR $(95 \% \mathrm{Cl})$ & $p$-value & $\begin{array}{c}\mathrm{P} \\
(\%)\end{array}$ & PR $(95 \% \mathrm{Cl})$ & $\mathrm{p}$-value \\
\hline $\begin{array}{l}\text { Preterm birth (according } \\
\text { to LMP) }\end{array}$ & & & $0.002^{*}$ & & & $0.2^{*}$ & & & $0.5^{*}$ \\
\hline Yes & 70.1 & $1.10(1.04 ; 1.10)$ & & 64.3 & 1 & & 53.9 & 1 & \\
\hline No & 63.8 & 1 & & 66.9 & $1.04(0.98 ; 1.11)$ & & 55.5 & $1.03(0.95 ; 1.11)$ & \\
\hline Firstborn child & & & $<0.001^{*}$ & & & $<0.001^{*}$ & & & $0.001^{*}$ \\
\hline Yes & 69.9 & $1.13(1.08 ; 1.18)$ & & 70.1 & $1.16(1.10 ; 1.21)$ & & 57.8 & $1.10(1.04 ; 1.16)$ & \\
\hline No & 61.9 & 1 & & 60.6 & 1 & & 52.6 & 1 & \\
\hline Type of delivery & & & $0.002^{*}$ & & & $<0.001^{*}$ & & & $<0.001^{*}$ \\
\hline C-section & 67.6 & $1.07(1.03 ; 1.12)$ & & 68.6 & $1.13(1.08 ; 1.18)$ & & 59.5 & $1.18(1.11 ; 1.24)$ & \\
\hline Vaginal & 62.8 & 1 & & 60.8 & 1 & & 50.6 & 1 & \\
\hline Gestational morbidity & & & $<0.001^{*}$ & & & $0.3^{*}$ & & & $0.01^{*}$ \\
\hline Yes & 66.5 & $1.13(1.06 ; 1.20)$ & & 64.8 & $1.03(0.97 ; 1.10)$ & & 55.6 & $1.10(1.02 ; 1.19)$ & \\
\hline No & 59.1 & 1 & & 62.8 & 1 & & 50.4 & 1 & \\
\hline Hospitalization & & & $<0.001^{*}$ & & & $0.4^{*}$ & & & $0.02 *$ \\
\hline Yes & 81.0 & $1.27(1.19 ; 1.35)$ & & 67.8 & $1.04(0.95 ; 1.13)$ & & 57.4 & $1.13(1.02 ; 1.26)$ & \\
\hline No & 63.9 & 1 & & 63.6 & 1 & & 54.4 & 1 & \\
\hline $\begin{array}{l}\text { Mother's perception of } \\
\text { their child's health }\end{array}$ & & & - & & & $<0.001$ ** & & & $<0.001 * *$ \\
\hline Excellent & - & - & & 58.0 & 1 & & 45.3 & 1 & \\
\hline Very good & - & - & & 67.9 & $1.17(1.10 ; 1.25)$ & & 54.8 & $1.21(1.11 ; 1.32)$ & \\
\hline Good & - & - & & 66.8 & $1.15(1.09 ; 1.22)$ & & 59.1 & $1.30(1.21 ; 1.40)$ & \\
\hline Fair/Poor & - & - & & 80.6 & $1.39(1.29 ; 1.50)$ & & 72.1 & $1.59(1.45 ; 1.74)$ & \\
\hline Breastfeeding (3 months) & & & $<0.001$ * * & & & - & & & - \\
\hline Never breastfed & 82.2 & $1.32(1.20 ; 1.45)$ & & - & - & & - & - & \\
\hline Quit breastfeeding & 71.2 & $1.14(1.09 ; 1.20)$ & & - & - & & - & - & \\
\hline Currently breastfeeding & 62.4 & 1 & & - & - & & - & - & \\
\hline $\begin{array}{l}\text { Breastfeeding (12 and } 24 \\
\text { months) }\end{array}$ & & & - & & & $0.6^{*}$ & & & $0.9^{*}$ \\
\hline Never breastfed & - & - & & 62.2 & $0.98(0.84 ; 1.13)$ & & 58.3 & $1.07(0.91 ; 1.26)$ & \\
\hline $\begin{array}{l}\text { Breastfeeding up to } 3 \\
\text { months }\end{array}$ & - & - & & 66.0 & $1.04(0.98 ; 1.10)$ & & 54.4 & $1.00(0.93 ; 1.07)$ & \\
\hline $\begin{array}{l}\text { Breastfeeding } 3 \text { to } 6 \\
\text { months }\end{array}$ & - & - & & 65.1 & $1.02(0.96 ; 1.09)$ & & 54.7 & $1.00(0.93 ; 1.08)$ & \\
\hline $\begin{array}{l}\text { Breastfeeding longer } \\
\text { than } 6 \text { months }\end{array}$ & - & - & & 63.6 & 1 & & 54.6 & 1 & \\
\hline
\end{tabular}

ICU: Intensive care unit

IEN: National Economic Index (quintiles for the city of Pelotas)

LMP: Last menstrual period

a Data collected at the follow-up of 12 months, $\mathrm{N}=3,907$

* Wald's test for heterogeneity

** Wald's test for linear trend

use seen among better-off individuals..$^{2,11,19}$ In our study, socioeconomic condition did not affect medicine use at any of the follow-up times. Although socioeconomic condition was associated to the outcome in the crude analysis, after controlling for confounders the effect was no longer observed, probably due to maternal education level and skin color.

Maternal education was positively associated to medicine use at the three follow-up times and this association increased with the child's age. Another study from Pelotas (1982 Birth Cohort) showed that children from mothers with more than six years of education used $20 \%$ more medicines than those whose mothers had less than three years of schooling. ${ }^{9}$ It may be explained by greater confidence and knowledge of these mothers to purchase over-the-counter medicines. According to a study about inequalities in access and utilization of Brazilian health services, ${ }^{18}$ overall, higher education is associated to a better understanding of the health-disease process. 
Table 2. Adjusted analysis ${ }^{\mathbf{a}}$ of factors associated to medicine use at 3,12 and 24 months of age. 2004 Pelotas Birth Cohort, Southern Brazil.

\begin{tabular}{|c|c|c|c|c|c|c|c|}
\hline \multirow{2}{*}{ Variable } & \multicolumn{2}{|c|}{3 months } & \multicolumn{2}{|c|}{12 months } & \multicolumn{2}{|c|}{24 months } & \multirow{2}{*}{$\begin{array}{l}\text { Level of } \\
\text { Analysis }\end{array}$} \\
\hline & $\mathrm{PR}(95 \% \mathrm{Cl})$ & $p$-value & PR $(95 \% \mathrm{Cl})$ & p-value & PR $(95 \% \mathrm{Cl})$ & p-value & \\
\hline Gender & & $0.4^{*}$ & & $0.03 *$ & & $0.1^{*}$ & 1 \\
\hline Male & $0.98(0.94 ; 1.03)$ & & $1.05(1.00 ; 1.10)$ & & $1.05(0.99 ; 1.11)$ & & \\
\hline Female & 1 & & 1 & & 1 & & \\
\hline $\begin{array}{l}\text { Maternal skin color (as } \\
\text { observed by the interviewer) }\end{array}$ & & $0.04^{*}$ & & $0.09^{*}$ & & $0.09 *$ & 1 \\
\hline White & $1.09(1.02 ; 1.16)$ & & $1.02(0.96 ; 1.09)$ & & $1.10(1.01 ; 1.19)$ & & \\
\hline Black & 1 & & 1 & & 1 & & \\
\hline Other & $1.06(0.96 ; 1.18)$ & & $0.90(0.80 ; 1.02)$ & & $1.07(0.94 ; 1.23)$ & & \\
\hline Maternal age (complete years) & & $0.01 * *$ & & $0.1 * *$ & & $0.7^{*}$ & 1 \\
\hline $12-19$ & $1.13(1.07 ; 1.24)$ & & $1.13(1.03 ; 1.26)$ & & $1.08(0.95 ; 1.21)$ & & \\
\hline $20-29$ & $1.07(0.98 ; 1.17)$ & & $1.10(1.01 ; 1.20)$ & & $1.06(0.96 ; 1.18)$ & & \\
\hline $30-35$ & $1.06(0.97 ; 1.17$ & & $1.14(1.04 ; 1.26)$ & & $1.06(0.94 ; 1.18)$ & & \\
\hline$\geq 36$ & 1 & & 1 & & 1 & & \\
\hline $\begin{array}{l}\text { Socioeconomic level (quintiles } \\
\text { of IEN) }\end{array}$ & & $0.09^{* *}$ & & $0.06^{* *}$ & & $0.48^{* *}$ & 1 \\
\hline $1 \mathrm{st}$ & 1 & & 1 & & 1 & & \\
\hline $2 n d$ & $1.11(1.03 ; 1.20)$ & & $1.11(1.03 ; 1.20)$ & & $1.09(0.99 ; 1.20)$ & & \\
\hline $3 r d$ & $1.09(1.01 ; 1.18)$ & & $1.08(0.99 ; 1.17)$ & & $1.11(1.01 ; 1.23)$ & & \\
\hline 4 th & $1.09(1.01 ; 1.19)$ & & $1.10(1 ; 1.20)$ & & $1.07(0.96 ; 1.19)$ & & \\
\hline 5th & $1.09(1 ; 1.19)$ & & $1.11(1.01 ; 1.22)$ & & $1.05(0.94 ; 1.18)$ & & \\
\hline $\begin{array}{l}\text { Maternal education (complete } \\
\text { (years of schooling) }\end{array}$ & & $0.04^{* *}$ & & $0.05^{* *}$ & & $<0.001^{* *}$ & 1 \\
\hline $0-4$ & 1 & & 1 & & 1 & & \\
\hline $5-8$ & $1.02(0.94 ; 1.10)$ & & $1.03(0.95 ; 1.11)$ & & $1.22(1.10 ; 1.36)$ & & \\
\hline $9-11$ & $1.09(1.01 ; 1.18)$ & & $1.08(0.99 ; 1.17)$ & & $1.29(1.16 ; 1.44)$ & & \\
\hline$\geq 12$ & $1.07(0.96 ; 1.19)$ & & $1.09(0.97 ; 1.21)$ & & $1.34(1.18 ; 1.53)$ & & \\
\hline Mother with health insurance & & $0.1^{*}$ & & $0.02^{*}$ & & $<0.001^{*}$ & 1 \\
\hline Yes & $1.04(0.99 ; 1.10)$ & & $1.08(1.01 ; 1.15)$ & & $1.13(1.06 ; 1.21)$ & & \\
\hline No & 1 & & 1 & & 1 & & \\
\hline Child with health insurance & & - & & $0.006^{*}$ & & $0.3^{*}$ & 1 \\
\hline Yes & - & & $1.09(1.02 ; 1.16)$ & & $1.04(0.97 ; 1.13)$ & & \\
\hline No & - & & 1 & & 1 & & \\
\hline Intrapartum complications & & $0.009^{*}$ & & $0.1^{*}$ & & $0.9^{*}$ & 2 \\
\hline Yes & $1.10(1.02 ; 1.18)$ & & $1.06(0.98 ; 1.14)$ & & $1.01(0.89 ; 1.14)$ & & \\
\hline No & 1 & & 1 & & 1 & & \\
\hline Neonatal ICU admission & & $0.2^{*}$ & & $0.9^{*}$ & & $0.01 *$ & 2 \\
\hline Yes & $1.08(0.96 ; 1.22)$ & & $1.01(0.89 ; 1.16)$ & & $1.16(1.03 ; 1.30)$ & & \\
\hline No & 1 & & 1 & & 1 & & \\
\hline Low birthweight (below 2500 g) & & $0.01^{*}$ & & $0.1^{*}$ & & $0.2^{*}$ & 2 \\
\hline Yes & $1.11(1.02 ; 1.21)$ & & $1.07(0.99 ; 1.16)$ & & $1.07(0.95 ; 1.20)$ & & \\
\hline No & 1 & & 1 & & 1 & & \\
\hline $\begin{array}{l}\text { Preterm birth (according to } \\
\text { LMP) }\end{array}$ & & $0.09 *$ & & $0.6^{*}$ & & $0.6^{*}$ & 2 \\
\hline Yes & $1.06(0.99 ; 1.13)$ & & 1 & & 1 & & \\
\hline No & 1 & & $1.02(0.95 ; 1.09)$ & & $1.02(0.93 ; 1.12)$ & & \\
\hline
\end{tabular}

To be continued 
Table 2 continuation

\begin{tabular}{|c|c|c|c|c|c|c|c|}
\hline \multirow{2}{*}{ Variable } & \multicolumn{2}{|c|}{3 months } & \multicolumn{2}{|c|}{12 months } & \multicolumn{2}{|c|}{24 months } & \multirow{2}{*}{$\begin{array}{l}\text { Level of } \\
\text { Analysis }^{\mathbf{b}}\end{array}$} \\
\hline & $\mathrm{PR}(95 \% \mathrm{Cl})$ & p-value & $\mathrm{PR}(95 \% \mathrm{Cl})$ & p-value & $\mathrm{PR}(95 \% \mathrm{Cl})$ & p-value & \\
\hline Firstborn child & & $0.001 *$ & & $<0.001^{*}$ & & $0.3^{*}$ & 2 \\
\hline Yes & $1.10(1.04 ; 1.16)$ & & $1.10(1.04 ; 1.16)$ & & $1.03(0.97 ; 1.10)$ & & \\
\hline No & 1 & & 1 & & 1 & & \\
\hline Type of delivery & & $0.3^{*}$ & & $0.06^{*}$ & & $0.007^{*}$ & 2 \\
\hline Cesarean & $1.02(0.97 ; 1.08)$ & & $1.05(1.00 ; 1.10)$ & & $1.09(1.02 ; 1.16)$ & & \\
\hline Vaginal & 1 & & 1 & & 1 & & \\
\hline Gestational morbidity & & $<0.001^{*}$ & & $0.2^{*}$ & & $0.006^{*}$ & 2 \\
\hline Yes & $1.16(1.08 ; 1.24)$ & & $1.04(0.98 ; 1.11)$ & & $1.12(1.03 ; 1.21)$ & & \\
\hline No & 1 & & 1 & & 1 & & \\
\hline Hospitalization & & $<0.001^{*}$ & & $1.0^{*}$ & & $0.1^{*}$ & 3 \\
\hline Yes & $1.26(1.18 ; 1.35)$ & & $1.00(0.91 ; 1.10)$ & & $1.09(0.99 ; 1.21)$ & & \\
\hline No & 1 & & 1 & & 1 & & \\
\hline $\begin{array}{l}\text { Mother's perception of their } \\
\text { child's health }^{\mathbf{c}}\end{array}$ & & - & & $<0.001 * *$ & & $<0.001 * *$ & 3 \\
\hline Excellent & - & & 1 & & 1 & & \\
\hline Very good & - & & $1.16(1.09 ; 1.24)$ & & $1.18(1.08 ; 1.29)$ & & \\
\hline Good & - & & $1.23(1.16 ; 1.30)$ & & $1.39(1.29 ; 1.50)$ & & \\
\hline Fair/Poor & - & & $1.52(1.40 ; 1.64)$ & & $1.74(1.58 ; 1.91)$ & & \\
\hline Breastfeeding (3 months) & & $<0.001 * *$ & - & - & - & - & 3 \\
\hline Never breastfed & $1.25(1.13 ; 1.38)$ & & - & & - & & \\
\hline Quit breastfeeding & $1.12(1.06 ; 1.18)$ & & - & & - & & \\
\hline Currently breastfeeding & 1 & & - & & - & & \\
\hline Breastfeeding (12 e 24 months) & - & - & & $0.2^{*}$ & & $1.0 *$ & 3 \\
\hline Never breastfed & - & & $0.88(0.74 ; 1.04)$ & & $1.00(0.84 ; 1.19)$ & & \\
\hline $\begin{array}{l}\text { Breastfeeding up to } 3 \\
\text { months }\end{array}$ & - & & $1.04(0.98 ; 1.11)$ & & $1.00(0.92 ; 1.06)$ & & \\
\hline Breastfeeding 3 to 6 months & - & & $1.01(0.94 ; 1.07)$ & & $0.98(0.91 ; 1.06)$ & & \\
\hline $\begin{array}{l}\text { Breastfeeding longer than } 6 \\
\text { months }\end{array}$ & - & & 1 & & 1 & & \\
\hline
\end{tabular}

ICU: Intensive care unit

IEN: National Economic Index (quintiles for the city of Pelotas)

LMP: Last menstrual period

${ }^{a}$ Variables with p-values between $5 \%$ and $20 \%$ were retained in the model to control for confounders. Those with p-values above $20 \%$ were excluded from the model.

b All variables are controlled for the same level and upper level.

${ }^{c}$ Data collected at the follow-up of 12 months, $\mathrm{N}=3,907$

* Wald's test for heterogeneity

** Wald's test for linear trend

Maternal age was negatively associated to medicine use at three months of age. We did not find any studies that evaluated similar associations, however, the literature shows that infants fathered by teenagers ( $<20$ years old) had an increased risk of preterm birth, low birthweight, small-for-gestational-age births, low Apgar score, neonatal mortality and post-neonatal mortality. ${ }^{14}$ Considering that teenage parents carry an increased risk of adverse birth outcomes, regardless of maternal confounders, it is expect greater medicine use, especially in early childhood.

Children from white compared to those from black mothers had a slightly higher medicine use, and, at three months, this association remained after controlling for maternal education and socioeconomic condition. Similar use patterns were reported in a study with children (4-11 years old) from Salvador, Northeastern Brazil. ${ }^{24}$ In another study among adults, although the highest prevalence was reported in white individuals, skin color was not associated to medicine use after adjustment. ${ }^{10}$

Maternal and/or child's health insurance positively influenced medicine use after 12 months of age. This fact may be associated to higher access to health care services and thus higher number of medical visits. ${ }^{20}$ Two other studies, one from Cataluña (Spain) about factors associated to medicine use during childhood, ${ }^{21}$ and the other a Brazilian study, ${ }^{2}$ showed the same association between medicine use and health insurance. 
Being a firstborn child was associated to higher use at three and 12 months of age. The same trend was observed in previous birth cohorts from Pelotas (1982 and 1993), probably due to family's anxiety and concerns about the child's health. ${ }^{9,28}$

Different variables directly or indirectly related to more severe health conditions (neonatal ICU care, hospitalization, low birthweight or gestational conditions), as well as the mother's perception of their child's health were associated to a higher prevalence of medicine use. These variables contribute to the understanding of the relations found as they all point to the same direction and are consistently associated.

A study with 3-month-olds showed that children hospitalized in the previous 90 days were more likely to use medicines (adjusted OR=2.34) ${ }^{28}$ Another study showed an increased risk of medicine use $(\mathrm{PR}=1.98)$ among children (4 to 11 years old) presenting health problems in the previous two weeks. ${ }^{24}$

Higher medicine use among low birthweight children is not surprising considering the amount of evidence linking low birthweight to health problems throughout life, and especially during childhood. ${ }^{7,8}$ Preterm birth was not associated to medicine use after controlling for confounders, probably due to simultaneous control for low birthweight.

Gestational morbidities may affect child's health. In a study about hypertension and gestational diabetes, two of the most prevalent gestational conditions, the authors observed that the presence of one or both of these conditions was also associated with adverse consequences for the mother and infant (both acute and long-term). ${ }^{26}$ Although we did not find any studies associating gestational morbidities and medicine use among children, higher use seen in our study may be explained by health problems resulting from gestational morbidities.

The mother's perception of the child's health as fair or poor was a strong predictor of medicine use, and the older the child, the greater this association. It can possibly be explained by the same reasons behind higher medicine use among children from highly educated mothers. This finding is in agreement with studies carried out in Spain, Brazil and Sweden. ${ }^{10,21,24}$

\section{REFERENCES}

1. Abajo FJ. El medicamento como solución y como problema para la salud pública. Una breve incursión a los objetivos de la farmacoepidemiología. Rev Esp Salud Publica. 2001;75(4):281-4.

2. Arrais PSD, Brito LL, Barreto ML, Coelho HLL. Prevalência e fatores determinantes do consumo de medicamentos no Município de Fortaleza, Ceará, Brasil. Cad Saude Publica. 2005;21(6):1737-46. DOI:10.1590/S0102-311X2005000600021
At three months, children who were never breastfed used $25 \%$ more medicines than those who were still being breastfed. The 1982 Pelotas Cohort study showed that children who were not exclusively breastfed were twice more likely to use medicines compared to those exclusively breastfed. According to this study, mothers exclusively breastfeeding their children are apparently more confident and less anxious, and their children have better health status which would reduce medicine use. ${ }^{28}$

Among the limitations of this study recall bias should be considered although the authors sought to overcome it by using a 15-day recall period. Potential misinformation regarding the medicines used by the children based on their mothers' reports was prevented by asking them to bring in medicine packages and prescriptions (in the three follow-up times, more than $55 \%$ of medicines packages were shown).

The aspects involving medicine use in children discussed here are best understood by socioeconomic, perinatal and behavioral characteristics that cannot be analyzed separately as they are all linked to the outcome.

Maternal schooling is a factor that can be changed by interventions as it influences medicine use. Potential education interventions among mothers about medicine use in children during childhood may promote a more rational medicine use in early life.

Epidemiological studies about medicine use in children have focused on the investigation of the most used pharmacological classes and determinants of usage, but little has been explored about perinatal characteristics that influence medicine use during early life. Medicine is an important health determinant and to study use patterns may help preventing inadequate use among infants.

Different variables influence medicine use during the first two years of life and it changes as the child grows. Most associated factors were somehow related to maternal factors and all the remaining were related to child's health problems. Medication use in children is indicated when following scientific guidelines, but we recognize that, especially at younger ages, social concerns may have a stronger influence than medical reasons.
3. Barros AJD, Hirakata VN. Alternatives for logistic regression in cross-sectional studies: an empirical comparison of models that directly estimate the prevalence ratio. BMC Med Res Methodol. 2003;3:21. DOI:10.1186/1471-2288-3-21

4. Barros AJD, Victora CG. Indicador econômico para o Brasil baseado no censo demográfico de 2000. Rev Saude Publica. 2005;39(4):523-9. DOI:10.1590/ S0034-89102005000400002 
5. Barros AJD, Santos IS, Victora CG, Albernaz EP, Domingues MR, Timm IK, et al. The 2004 Pelotas birth cohort: methods and description. Rev Saude Publica. 2006;40(3):402-13. DOI:10.1590/S003489102006000300007

6. Barros FC, Victora CG, Matijasevich A, Santos IS, Horta $\mathrm{BL}$, Silveira MF, et al. Preterm births, low birth weight, and intrauterine growth restriction in three birth cohorts in Southern Brazil: 1982, 1993 and 2004. Cad Saude Publica. 2008;24 Suppl 3:s390-8. DOI:10.1590/ S0102-311X2008001500004

7. Barker DJ, Osmond C. Infant mortality, childhood nutrition, and ischaemic heart disease in England and Wales. Lancet. 1986;1(8489):1077-81. DOI:10.1016/ S0140-6736(86)91340-1

8. Barker DJ, Gluckman PD, Godfrey KM, Harding JE, Owens JA, Robinson JS. Fetal nutrition and cardiovascular disease in adult life. Lancet. 1993;341(8850):938-41. DOI:10.1016/01406736(93)91224-A

9. Béria JU, Victora CG, Barros FC, Teixeira AB, Lombardi C. Epidemiologia do consumo de medicamentos em crianças de centro urbano da região sul do Brasil. Rev Saude Publica. 1993;27(2):95-104. DOI:10.1590/ S0034-89101993000200004

10. Bertoldi AD, Barros AJD, Hallal PC, Lima RC. Utilização de medicamentos em adultos: prevalência e determinantes individuais. Rev Saude Publica. 2004;38(2):228-38. DOI:10.1590/S003489102004000200012

11. Bertoldi AD, Barros AJD, Wagner A, Ross-Degnan D, Hallal PC. Medicine access and utilization in a population covered by primary health care in Brazil. Health Policy. 2009;89(3):295-302. DOI:10.1016/j. healthpol.2008.07.001

12. Carrasco-Garrido $P$, Jiménez-García R, Hernández Barrera V, López de Andrés A, Gil de Miguel A. Medication consumption in the Spanish pediatric population: related factors and time-trend 1993-2003. Br J Clin Pharmacol. 2009;68(3):455-61. DOI:10.1111/ j.1365-2125.2009.03449.x

13. Centers for Disease Control and Prevention. Epi Info 604d. Atlanta; 2001.

14. Chen XK, Wen SW, Krewski D, Fleming N, Yang Q, Walker MC. Paternal age and adverse birth outcomes: teenager or 40+, who is at risk? Hum Reprod. 2008;23(6):1290-6. DOI:10.1093/humrep/dem403

15. Clavenna A, Sequi M, Bortolotti A, Merlino L, Fortino I, Bonati M. Determinants of the drug utilization profile in the paediatric population in Italy's Lombardy Region. Br J Clin Pharmacol. 2009;67(5):565-71. DOI:10.1111/j.1365-2125.2009.03380.x

16. Hardon A, Hodkin C, Fresle D. Cómo investigar el uso de medicamentos por parte de los consumidores.
Geneva: Organización Mundial de la Salud; Universidad de Amsterdam; 2004.

17. Hawkins N, Golding J. A survey of the administration of drugs to young infants. The Alspac Survey Team. Avon Longitudinal Study of Pregnancy and Childhood. Br J Clin Pharmacol. 1995;40(1):79-82.

18. Lima JC, Azoury EB, Bastos LHCV, Coutinho MM, Pereira NN, Ferreira SCC. Desigualdades no acesso e utilização de serviços de saúde no Brasil. Saude Debate. 2002;26(60):62-70.

19. Loyola Filho Al, Uchoa E, Guerra HL, Firmo JOA, Lima-Costa MF. Prevalência e fatores associados à automedicação: resultados do projeto Bambuí. Rev Saude Publica. 2002;36(1):55-62. DOI:10.1590/ S0034-89102002000100009

20. Mendoza-Sassi R, Béria JU, Barros AJD. Outpatient health service utilization and associated factors: a population-based study. Rev Saude Publica. 2003;37(3):372-8. DOI:10.1590/S003489102003000300017

21. Rajmil L, Ruiz C, Segú JL, Fernández E, Segura A. Factores asociados al consumo de medicamentos en la población infantil. Med Clin (Barc). 2000;114(6):214-7.

22. Rylance GW, Woods CG, Cullen RE, Rylance ME. Use of drugs by children. BMJ. 1988;297(6646):445-7.

23. Sans S, Paluzie G, Puiga T, Balañá L, BalaguerVintró I. Prevalencia del consumo de medicamentos en la población adulta de Cataluña. Gac Sanit. 2002;16(2):121-30. DOI:10.1590/S021391112002000200005

24. Santos DB, Barreto ML, Coelho HL. Drug use and associated factors in children living in poor areas. Rev Saude Publica. 2009;43(5):768-78. DOI:10.1590/ S0034-89102009000500005

25. Schirm E, van den Berg P, Gebben H, Sauer P, De Jongvan den Berg L. Drug use of children in the community assessed through pharmacy dispensing data. $\mathrm{Br} / \mathrm{Clin}$ Pharmacol. 2000;50(5):473-8. DOI:10.1046/j.13652125.2000.00275.x

26. Sibaj BM, Ross MG. Hypertension in gestational diabetes mellitus: pathophysiology and long-term consequences. J Matern Fetal Neonatal Med. 2010;23(3):229-33. DOI:10.3109/14767050903550899

27. Victora CG, Hutly SR, Fuchs SC, Olinto MTA. The role of conceptual frameworks in epidemiological analysis: a hierarchical approach. Int J Epidemiol. 1997;26(1):224-7.

28. Weiderpass E, Béria JU, Barros FC, Victora CG, Tomasi E, Halpern R. Epidemiologia do consumo de medicamentos no primeiro trimestre de vida em centro urbano do Sul do Brasil. Rev Saude Publica. 1998;32(4):335-44. DOI:10.1590/S003489101998000400005

This study is based on data from the 2004 Pelotas Birth Cohort carried out in the Graduate Program in Epidemiology at the Universidade Federal de Pelotas. The 2004 Pelotas Birth Cohort is currently sponsored by the Wellcome Trust Initiative through the "Major Awards for Latin America on Health Consequences of Population Change". Previous stages of the study were sponsored by the World Health Organization (WHO), National Program for Excellence Centers (PRONEX), National Council of Technological and Scientific Development (CNPq), the Brazilian Ministry of Health and Pastoral da Criança. The authors declare no conflicts of interests. 